\title{
Reabilitação bucal de sequela de lesão central de células gigantes em paciente pediátrico: relato de caso
}

\author{
Oral rehabilitation of sequel of central lesion of giant cells in pediatric patients: case report
}

\author{
Rehabilitación oral de la secuela de lesión central de células gigantes em pacientes \\ pediátricos: reporte de caso
}

Gabriela Fontan Britto Rapôso ${ }^{1 *}$, Isabelle Costa de Almeida Perciano1 ${ }^{1}$ Aleska Dias Vanderlei ${ }^{1-2}$, Camila Maria Beder Ribeiro Girish Panjwani ${ }^{1-3}$.

\section{RESUMO}

Objetivo: Apresentar o relato de um caso de reabilitação bucal de uma sequela de lesão central de células gigantes em paciente pediátrico, dada a escassez literária sobre o tema e sua importância no planejamento da reabilitação bucal para os profissionais da área da saúde. Detalhamento do caso: Acompanhamento do planejamento e confecção de uma prótese parcial removível provisória. Discussão: Em pacientes pediátricos esperam-se prejuízos estéticos, fonéticos e mastigatórios acentuados do edentulismo; estes podem ser amenizados ou extinguidos com a adesão ao tratamento protético e colaboração do paciente e seu responsável. Considerações finais: Dada a natureza do desenvolvimento infantil, periodicamente deverão ser realizados ajustes ou substituições protéticas de acordo com o crescimento. Tratamentos definitivos como implantes ou próteses definitivas deverão aguardar até a fase adulta.

Palavras-chave: Prótese Parcial Removível, Reabilitação Bucal, Odontopediatria.

\begin{abstract}
Objective: To present the report of a case of oral rehabilitation of a sequela of central giant cell lesion in a pediatric patient, given the literary shortage on the subject and its importance in the planning of oral rehabilitation for health professionals. Case detail: Follow-up of the planning and preparation of a provisional removable partial denture. Discussion: Pediatric patients are expected to have aesthetic, phonetic and masticatory damages accentuated by edentulism; these can be softened or extinguished by adherence to the prosthetic treatment and collaboration of the patient and his / her caregiver. Final Consideration: Given the nature of childhood development, prosthetic adjustments or replacements should be periodically performed according to growth. Definitive treatments like implants or definitive prostheses should wait until the adult stage.
\end{abstract}

Keywords: Denture, Partial, Removable, Mouth Rehabilitation, Pediatric Dentistry.

\section{RESUMEN}

Objetivo: Presentar el informe de un caso de rehabilitación oral de una secuela de lesión de células gigantes centrales en un paciente pediátrico, dada la escasez literaria sobre el tema y su importancia en la planificación de la rehabilitación oral para profesionales de la salud. Detalle del caso: Seguimiento de la planificación y preparación de una prótesis parcial removible temporal. Discusión: Se espera que los pacientes pediátricos presenten daños estéticos, fonéticos y masticatorios acentuados por el edentulismo; Estos pueden ablandarse o extinguirse mediante la adherencia al tratamiento protésico y la colaboración del paciente y su cuidador. Consideraciones finales: Dada la naturaleza del desarrollo infantil, los ajustes protésicos o los reemplazos deben realizarse periódicamente de acuerdo con el crecimiento. Los tratamientos definitivos como implantes o prótesis definitivas deben esperar hasta la etapa adulta.

Palabras clave: Dentadira Parcial Removible, Rehabilitación Bucal, Odontología Pediátrica.

${ }^{1}$ Centro Universitário Cesmac, Maceió-AL. *E-mail: gabrielaisabelaraposo@gmail.com

2Universidade Estadual Paulista Júlio de Mesquita Filho. São Paulo-SP.

${ }^{3}$ Universidade Estadual de Campinas. Campinas-SP. 


\section{INTRODUÇÃO}

Entende-se por reabilitação bucal a restauração de forma e função do sistema estomatognático através da utilização de materiais de maior resistência e durabilidade como resinas compostas, amálgamas ou próteses (RODRIGUES FV, et al., 2019). Estas últimas, por sua vez, se tornam uma opção para a reabilitação oral de pacientes com perdas dentárias extensas que, como consequência da ausência dentária, ficam sujeitos a distúrbios emocionais, sociais, estéticos e de funcionalidade (BRELAZ KLAT, et al., 2016; COELHO MQ, et al., 2016; VULIĆEVIĆ Z, et al., 2017).

Em especial, quando presente em crianças e adolescentes, o impacto da perda pode ser significativo pela diminuição da capacidade mastigatória, fonação e prejuízos de ordem nutricional, bem como também do ponto de vista estético e psicológico ao estimular a redução de autoestima e tornar difícil a integração social (AGOSTINHO ACMG, et al., 2015; BRELAZ KLAT, et al., 2016; VULIĆEVIĆ Z, et al., 2017).

O edentulismo é considerado um dos maiores agravos à saúde bucal. Projeta-se fortemente no público brasileiro e caracteriza-se pela ausência parcial ou total de dentes (FERRAZ NGG, et al., 2016; SILVA BCM, et al., 2017). É um desafio que cujos resultados da intervenção dependem da idade e do tipo de dente perdido (SOUSA JM, et al., 2012), muito associada à desigualdade social (SILVA BCM, et al., 2017).

Comumente, esses dentes são perdidos principalmente por cárie e doença periodontal (SOUZA JEA, et al., 2009; FARIAS-NETO A, et al., 2011; SOUSA JM, et al., 2012; PEIXOTO ATP, et al., 2015; FERRAZ NGG, et al., 2016; VILELA et al., 2016; JORGE et al., 2019), mas casos de traumatismo dentário e oclusal e parafunções também podem ser considerados como causas em segundo plano (SILVA BCM, et al., 2017), bem como mutilações faciais severas ou sequelas de lesões proliferativas neoplásicas e não neoplásicas nos maxilares (TRENTO CL, et al., 2009).

Pacientes portadores de lesões tumorais e/ou de crescimento substancial geralmente são submetidos à terapia cirúrgica visando remover toda a lesão com uma margem de segurança mínima de $1 \mathrm{~cm}$ em todas as direções para evitar o risco de reincidência (VALLE CN, et al., 2016). Ameloblastomas, Carcinoma Espinocelular e Tumores de Células Gigantes podem ser exemplos da necessidade de ressecção cirúrgica parcial ou remoções dentárias, respectivamente (TRENTO CL, et al., 2009; VALLE CN, et al., 2016; SILVA WSA, et al., 2019).

No entanto, em caso de ressecções mais extensas, além da deformidade estética esperada, complicações graves como alteração oclusal, limitação dos movimentos mandibulares e desvio mandibular podem diminuir a qualidade de vida de um indivíduo (CATUNDA IS, et al., 2012).

Em odontopediatria, a perda precoce desses elementos interfere no crescimento craniofacial das crianças e pode resultar na diminuição do comprimento do arco, migração dos dentes vizinhos ao espaço originado, desenvolvimento de hábitos deletérios e, consequentemente, alterações na função mastigatória, fonética e estética (GUIMARÃES CA e OLIVEIRA RCG, 2017; NÓBREGA ML, et al., 2018).

Levando isso em consideração, a reabilitação protética desses elementos é de extrema importância para o equilíbrio da face e dos arcos dentários (GUIMARÃES CA e OLIVEIRA RCG, 2017), visando devolver a integridade das arcadas ao restabelecer suas funções normais e evitar a instalação de hábitos nocivos e mal oclusões (BRELAZ KLAT, et al., 2016).

Diante do exposto, o objetivo deste trabalho é apresentar o relato de um caso de reabilitação bucal em virtude da sequela de uma lesão central de células gigantes em paciente pediátrico, dada a escassez literária sobre o tema e sua importância no planejamento da reabilitação bucal para os profissionais da área da saúde.

\section{DETALHAMENTO DO CASO}

Paciente do gênero feminino, 10 anos de idade, compareceu à Clínica Odontológica com queixa principal de necessidade de reabilitação oral de área edêntula na região anterior em mandíbula. Relatou que a perda foi sequela cirúrgica após remoção de Lesão Central de Células Gigantes quando tinha 7 anos, onde os germes dos dentes permanentes sucessores também foram removidos. 
O exame clínico intrabucal revelou a ausência dos incisivos permanentes inferiores, caninos e primeiro molar direito decíduos (Figura 1). Ao exame extraoral observou-se falta de suporte labial inferior.

Figura 1 - Exame intraoral.

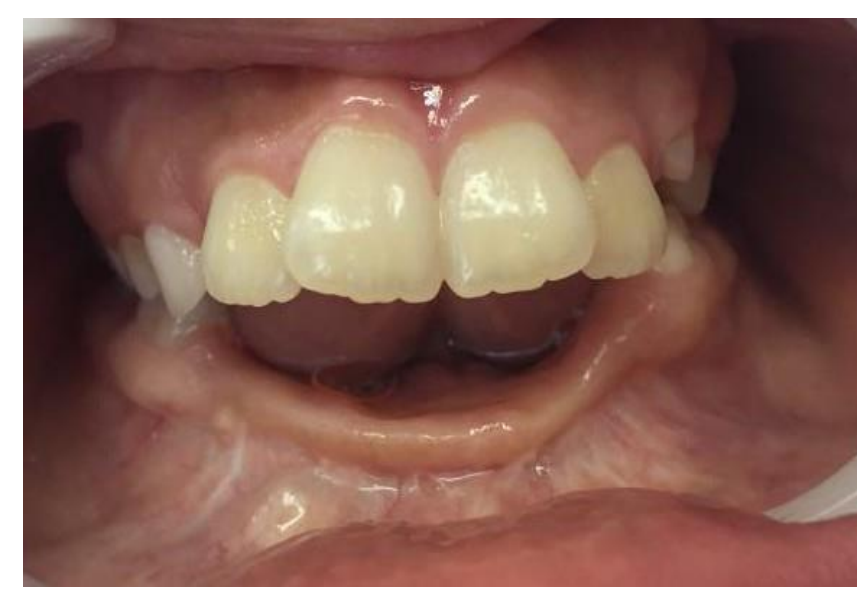

Fonte: Dados de pesquisa, 2019.

Na radiografia panorâmica anterior à cirurgia (Figura 2) foi observado acometimento de todos os germes permanentes, com exceção dos segundos molares, além do envolvimento das raízes do elemento 74 e raíz mesial das unidades 75 e 85 .

Figura 2 - Radiografia panorâmica antes da ressecção da lesão e suas proporções.

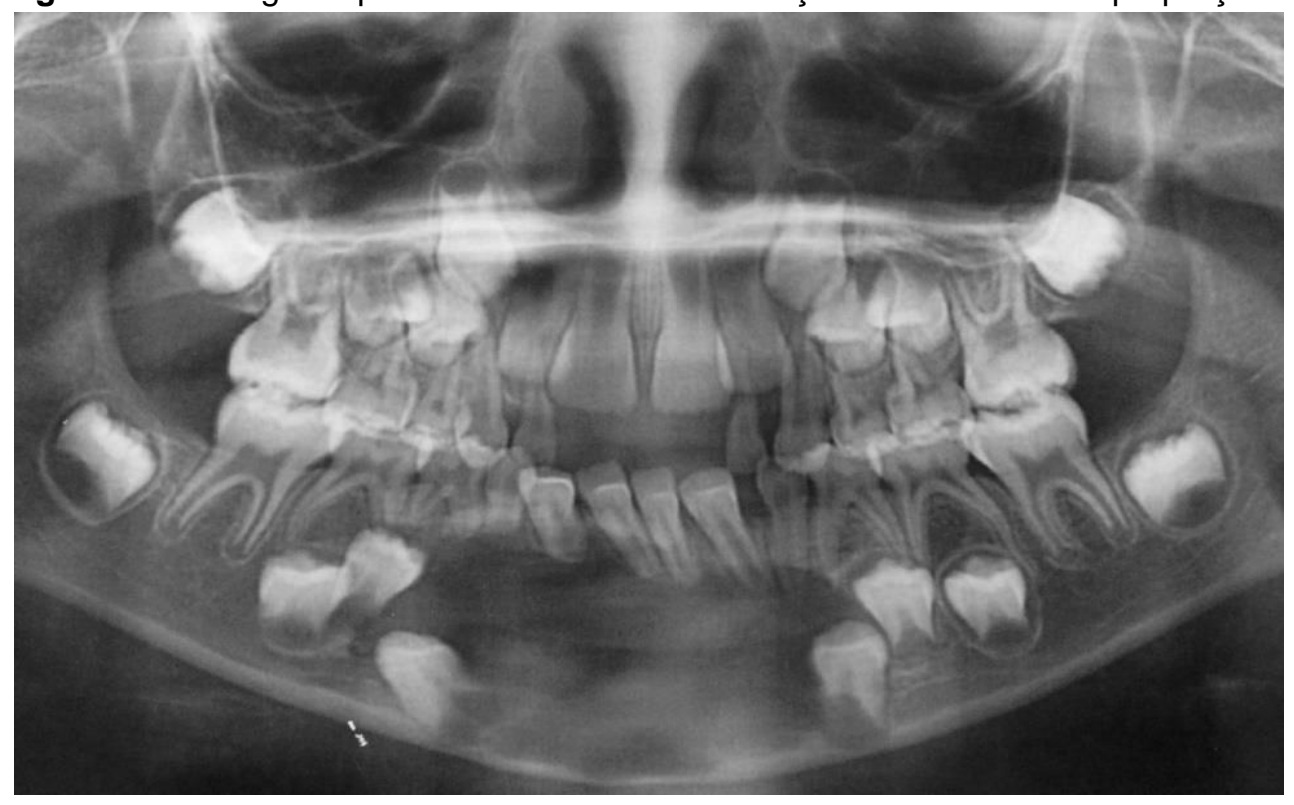

Fonte: Dados de pesquisa, 2019.

Após adequação do meio bucal, foi realizado o planejamento da reabilitação protética do paciente através de um modelo de estudo seguida da confecção da base de registro inferior, registro intermaxilar e montagem em articulador semi-ajustável (Figuras 3A, B e C). Dada à singularidade do caso, debateu-se a melhor opção do tratamento e, com isso, reabilitações definitivas como implantes acabaram sendo desprezadas pelo fato de a paciente ainda estar em desenvolvimento. Optou-se então pela confecção de uma prótese parcial removível provisória, levando em consideração trocas e/ou substituições da prótese até que a paciente atingisse a idade adulta, detalhes esses devidamente explicados ao responsável. 
Figura 3 - A. Planejamento da reabilitação. B. Montagem em articulador semi-ajustável, vista frontal. C. Montagem em articulador semi-ajustável, vista lateral.

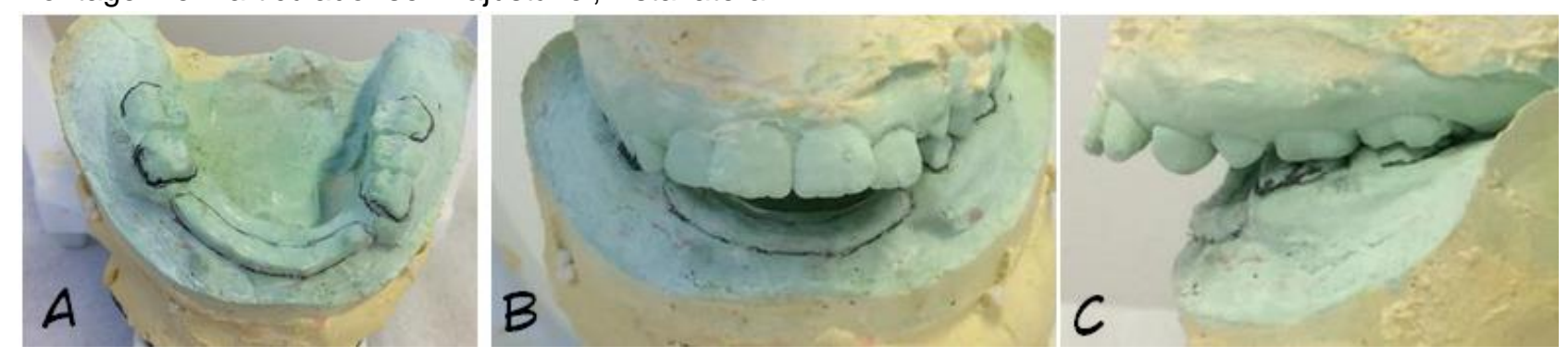

Fonte: Dados de pesquisa, 2019.

Através de um novo modelo de trabalho, foi realizada a montagem de dentes em cera (Figura 4 A e B) e prova da base, a qual se mostrou satisfatória para o caso, mesmo com a ausência da confecção dos nichos, por ser provisória (Figura 5 A, B e C). A prótese foi acrilizada utilizando fios ortodônticos para a sua retenção em boca, seguindo o modelo de um aparelho ortopédico (Figura 6).

Figura 4 - A. Vista frontal da montagem de dentes em cera no articulador semi-ajustável. B. Vista lateral da montagem de dentes em cera no articulador semi-ajustável.
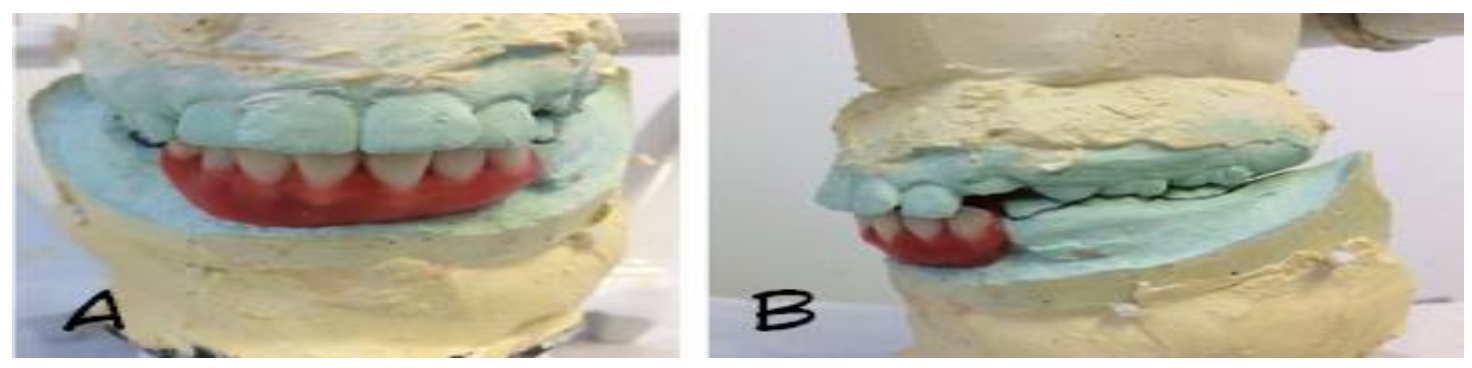

Fonte: Dados de pesquisa, 2019.

Figura 5 - A. Vista frontal da prova. B. Vista lateral. C. Vista oclusal.
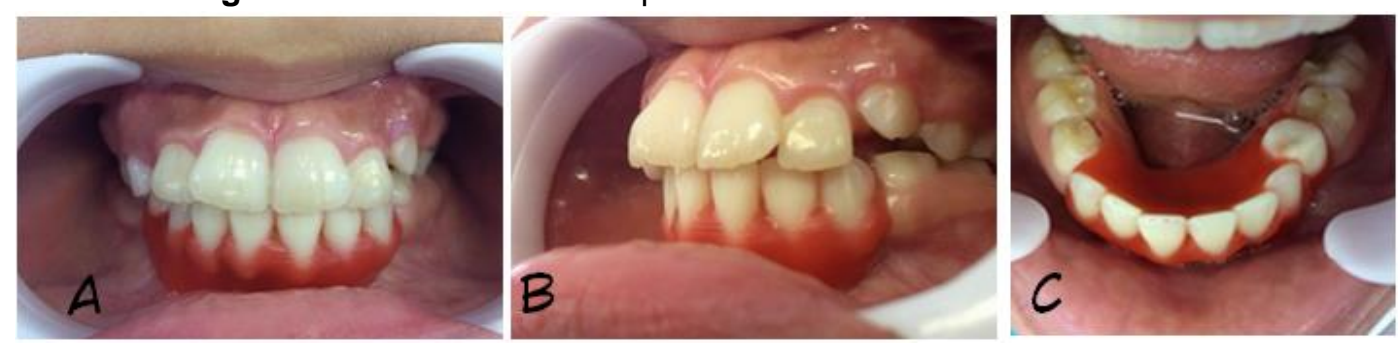

Fonte: Dados de pesquisa, 2019.

Figura 6 - Acrilização da prótese parcial removível.

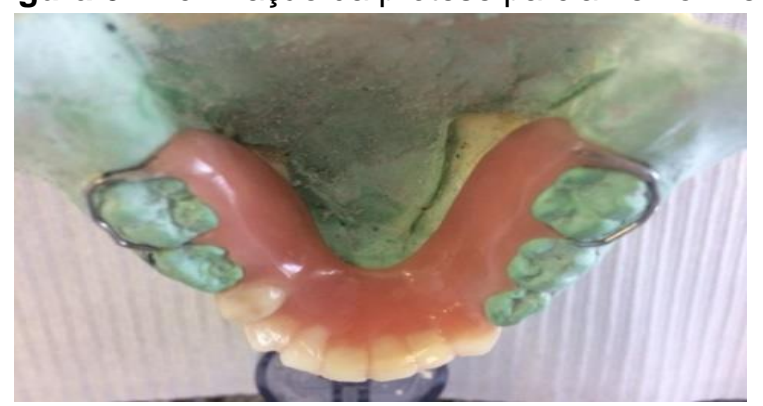

Fonte: Dados de pesquisa, 2019. 
Em seguida, ajustes oclusais foram confeccionados (Figura 7 A e B) e foram marcados controles para 24 horas, 7 dias, 14 dias, 1 mês, 3 meses, 6 meses e 12 meses para acompanhamento do desenvolvimento e assentamento concomitante. Ao fim, foram devolvidas estética, funcionalidade e autoestima para a paciente (Figura 8 A e B).

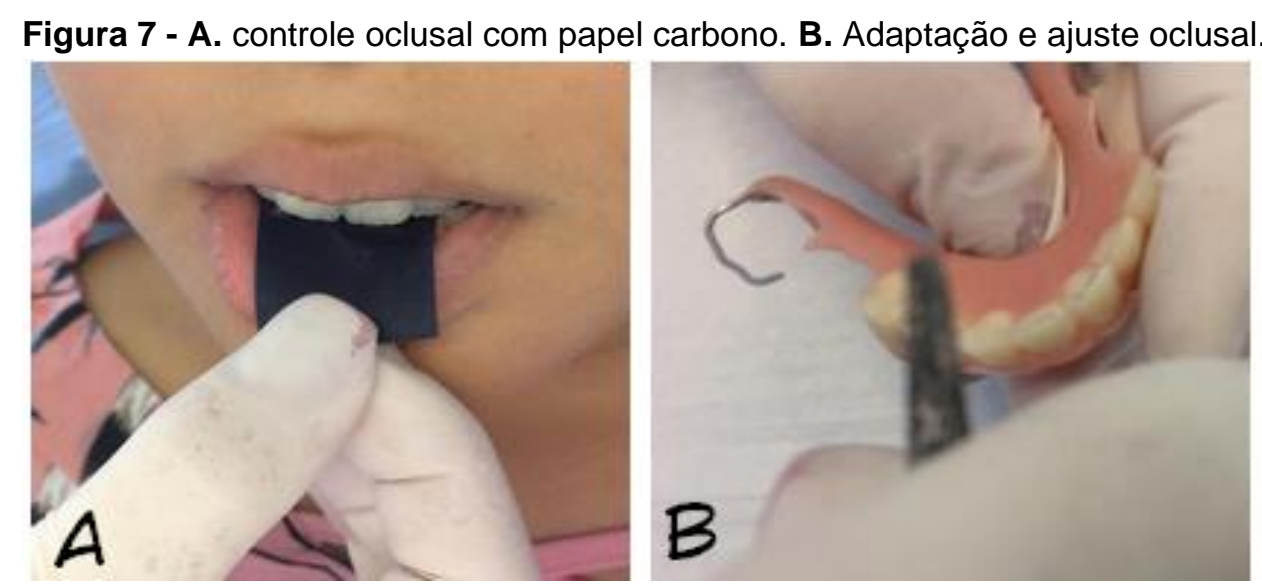

Fonte: Dados de pesquisa, 2019.

Figura 8 - Reabilitação satisfatória. A. boca entreaberta demonstrando os elementos reabilitados. B. Oclusão, funcionalidade e estética restituídas.

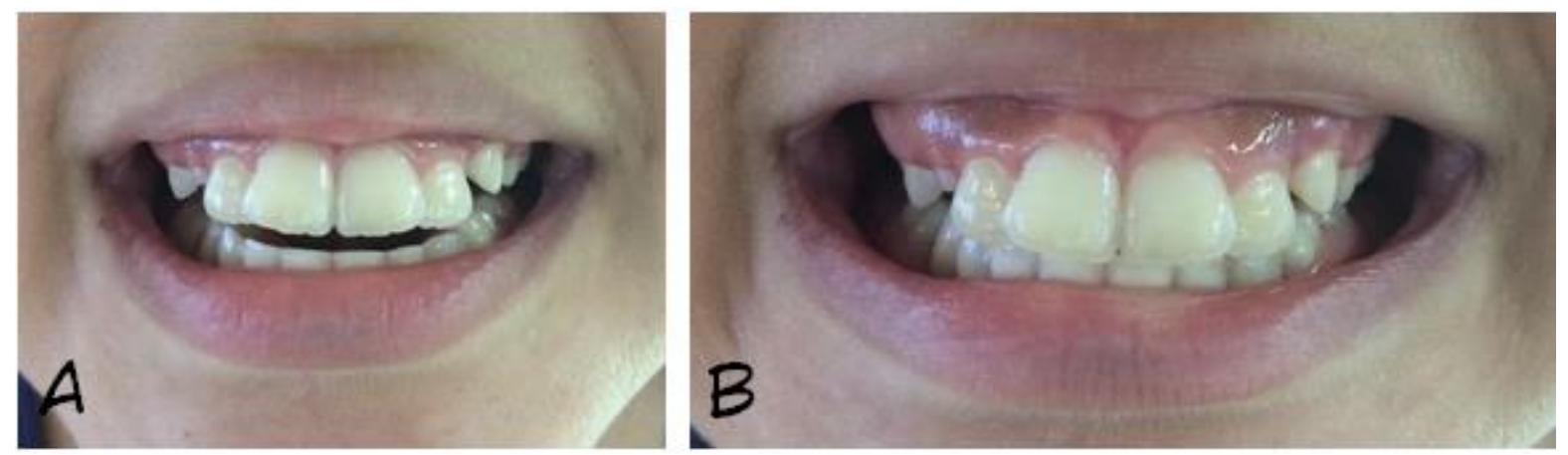

Fonte: Dados de pesquisa, 2019.

O acompanhamento dessa paciente deverá ser preferencialmente anual para acompanhar os picos de desenvolvimento e para realizar ajustes e/ou troca da prótese provisória de acordo. Estima-se que essa modalidade protética provisória continue até sua entrada na fase adulta, onde deverá realizar um tratamento fixo com implantes ou uma prótese definitiva corretamente planejada e assentada com um material mais resistente e duradouro.

\section{DISCUSSÃO}

A reabilitação protética de pacientes desdentados é versátil e pode ser realizada de diferentes maneiras a depender do julgo e competência profissional, necessidades, preferências, custo, higiene bucal e limitações biológicas (CAVALCANTI YW, et al., 2015; BRELAZ KLAT, et al., 2016).

Em odontopediatria, é indicada para a melhora das funções mastigatórias, fala e, também, para evitar traumas psicológicos decorrentes do prejuízo estético permeado pela falta dos dentes ao longo do crescimento (PEREIRA L e MIASATO JM, 2010; HERDT LOS, 2017; SALES PSM et al., 2018).

Comumente, a reposição protética desses elementos se dá através de mantenedores de espaço visando devolver a integridade das arcadas, reestabelecer funções normais de mastigação, deglutição, fonação e prevenir a instalação de hábitos nocivos e maloclusões até a erupção dos dentes permanentes, dado que a 
perda precoce influencie no desenvolvimento e, a depender do momento da perda tenha papel no não domínio da fala ou na aquisição de distúrbios transitórios até o período em que mecanismos compensatórios entrem em ação (PEREIRA L e MIASATO JM, 2010; BRELAZ KLAT, et al., 2016).

No entanto, em casos atípicos pediátricos como ressecções cirúrgicas que acometem dentes ou germe de dentes permanentes e podem causar mutilações faciais a depender do tamanho da área envolvida, diagnóstico e necessidade de margem de segurança, faz-se necessária uma reabilitação protética provisória contínua que acompanhe o período de desenvolvimento com modificações e/ou substituições até o final (RATHEE M, et al., 2016; SHIBAYAMA R, et al., 2016).

Nesse sentido, Próteses Parciais Removíveis (PPR) são consideradas uma opção de reestabelecimento estético, fonético e mastigatório de baixo custo, fácil confecção, de amplo acesso, (CAVALCANTI YW, et al., 2015; MARTINS MA, et al., 2016 AGUIAR ES, 2017), simples, conveniente e conservadora que permite alterações de acordo com o ritmo de crescimento (RATHEE M, et al., 2016).

No entanto, desafios em relação à correspondência de cor, tamanho e forma podem ser esperados, bem como modificações substanciais para alcançar uma estética aceitável (BRELAZ KLAT, et al., 2016; MARTINS MA, et al., 2016) e insatisfação do paciente com a estrutura metálica, além da preocupação do equilíbrio da distribuição de forças para manter o rebordo alveolar e os dentes remanescentes em bom estado de saúde (AGUIAR ES, 2017).

A reabilitação de pacientes edêntulos parcialmente é um desafio que exige habilidade e conhecimento técnico pelo dentista que deve atentar para guia anterior, redução da dimensão vertical oclusal e desfiguração oclusal (BRELAZ KLAT, et al., 2016).

A busca pelo correto relacionamento maxilomandibular é afetada pela perda dentária, a qual influencia diretamente o desequilíbrio do sistema estomatognático, resultando em alterações nas funções mastigatórias, fonéticas, estéticas, esqueléticas e de harmonização facial, além de dor, desgaste das estruturas articulares e estresse muscular (CAVALCANTI YW, et al., 2015).

$\mathrm{Na}$ dentição mista, o esperado é que a reabilitação mostre resultados de melhora na qualidade e eficiência mastigatória através da recuperação da posição normal da mandíbula delineada pela reposição dos dentes (DAINEZI VB, et al., 2015). Nesse caso, a paciente mostrou-se satisfeita e obteve restituição das funções primárias e estéticas e ajustes forem confeccionados visando esse fim, além de longevidade e durabilidade até a próxima substituição e/ou ajuste protético.

De modo geral, o sucesso do tratamento depende muito da cooperação da criança e seu responsável que deverão estar igualmente comprometidos e informados da necessidade de mudanças e um posterior tratamento reabilitador definitivo (RATHEE M, et al., 2016; JORGE CF, et al., 2019). No entanto, muitas vezes isso não acontece e a preocupação dos responsáveis com as crianças comumente aparece quando algum fator local desencadeia algum sintoma estressante como a dor ou um problema sistêmico (DAINEZI VB, et al., 2015).

Corroborando com Daizeni VB et al. (2015), Gomes MC et al. (2014) realizaram um estudo transversal com 843 pré-escolares e seus responsáveis, onde constataram que $32,1 \%$ das crianças sofriam com prevalência de impacto de condições de saúde bucal que afetavam sua qualidade de vida e apenas $26,2 \%$ das famílias tinham ciência, caracterizando-os com percepção ruim dos fatos.

Da mesma maneira, quando Castro FC et al. (2013) buscaram avaliar o impacto das condições clínicas bucais e fatores socioeconômicos na qualidade de vida das crianças à saúde bucal, encontram índices de $54,10 \%$ de cárie, ao que atribuíram à populações menos favorecidas de baixa renda com medo do tratamento e/ou falta de instrução. Em síntese, a reabilitação protética infantil é uma boa escolha para a melhora da qualidade de vida das crianças, mas é um passo que necessita acompanhamento constante e disciplina de todas as partes envolvidas no seu sucesso (DAINEZI VB, et al., 2015; RATHEE M, et al., 2016; AGUIAR ES, 2017; SALES PSM, et al., 2018; JORGE CF, et al., 2019). 


\section{CONSIDERAÇÕES FINAIS}

O tratamento protético reabilitador na infância é de extrema importância a depender da idade da perda dentária precoce. Prejuízos estéticos, fonéticos e mastigatórios causados pelo edentulismo parcial esperados, podem ser amenizados ou extinguidos com a adesão e colaboração do paciente e seu responsável. Com isso, além dos detalhes técnicos essenciais e fatores de colaboração, é necessário ter em mente que, ao optar pela reabilitação com próteses em odontopediatria, o paciente no qual elas foram instaladas deve ser monitorado para a realização de ajustes necessários ou substituição protética, dado o crescimento e desenvolvimento individual, para que mais tarde uma reabilitação definitiva seja executada.

\section{REFERÊNCIAS}

1. AGOSTINHO ACMG, et al. Edentulismo, uso de prótese e autopercepção de saúde bucal entre idosos. Revista de Odontologia da UNESP, São Paulo, 2015; 44(2): 74-79.

2. AGUIAR ES. Reabilitação oral através de prótese parcial removível: relato de caso. Monografia (Trabalho de Conclusão de Curso) - Universidade do Estado do Amazonas, Escola Superior de Ciências da Saúde, Manaus, 2017; 43 p.

3. BRELAZ KLAT, et al. Prótese parcial removível temporária em odontopediatria: relato de caso. Archives of Health Investigation, São Paulo, 2016; 5(1): 13-17.

4. CATUNDA IS, et al. Reconstrução mandibular com prótese de resina acrílica após ressecção de ameloblastoma. Relato de caso e avaliação da qualidade de vida. Revista de Cirurgia e Traumatologia Buco-Maxilo-Facial, Camaragibe, 2012; 12(4): 45-52.

5. CASTRO FC, et al. Impacto dos problemas bucais na qualidade de vida em pré-escolares. Pesquisa Brasileira em Odontopediatria e Clínica Integrada, Campina Grande, 2013; 13(4): 361-369.

6. CAVALCANTI YW, et al. Prótese parcial removível provisória tipo overlay na reabilitação oral de paciente com colapso oclusal posterior. Revista Brasileira de Ciências da Saúde, João Pessoa, 2015; 19(2): 143-150.

7. COELHO MQ, et al. Reabilitação bucal com próteses conjugadas - relato de caso. Full Dentistry in Science, São José dos Pinhais, 2016; 7(28): 64-69.

8. DAINEZI VB, et al. Reabilitação estética e funcional na primeira infância: relato de caso. Revista da Associação Paulista de Cirurgiões-Dentistas, São Paulo, 2015; 69(4): 387-393.

9. FARIAS-NETO A, et al. A prótese parcial removível no contexto da odontologia. Odontologia Clínico-Científica, Recife, 2011; 10(2): 125-128.

10. FERRAZ NGG, et al. Perdas dentais no atendimento de clínicas de atenção básica. Revista de Cirurgia e Traumatologia BucoMaxilo-Facial, Camaragibe, 2016; 16(1): 19-27.

11. GOMES MC, et al. Impact of oral health conditions on the quality of life of preschool children and their families: a cross-sectional study. Health and Quality of Life Outcomes, Nova lorque, 2014; 12(55): 01-12.

12. GUIMARÃES CA, OLIVEIRA RCG. Perda precoce de dentes decíduos. Relato de caso clínico. Revista Uningá Review, Maringá, 2017; 29(2): 28-33.

13. HERDT LOS. Reabilitação protética em paciente infantil com displasia ectodérmica. Monografia (Trabalho de Conclusão de Curso) - Universidade do Sul de Santa Catarina, Tubarão, 2017; 31 p.

14. JORGE CF, et al. O desafio do restabelecimento de um sorriso antiestético por meio de prótese fixa metal-free. Archives of Health Investigation, São Paulo, 2019; 8(1): 06-12.

15. MARTINS MA, et al. Prótese parcial removível com eixo rotacional. Investigação, São Paulo, 2016; 15(1): 114-117.

16. NÓBREGA ML, et al. Implicações da perda precoce em odontopediatria. Revista Pró-UniverSUS, Vassouras, $2018 ; 9(1): 61-67$.

17. PEIXOTO ATP, et al. Relação entre o uso de prótese removível e úlcera traumática - revisão de literatura. Journal of Oral Investigations, Rio de Janeiro, 2015; 4(1): 26-32.

18. PEREIRA L, MIASATO JM. Mantenedor de espaço estético-funcional em odontopediatria. Revista de Odontologia da Universidade da Cidade de São Paulo, São Paulo, 2010; 22(2): 154-162.

19. RATHEE M, et al. Early functional, esthetic, and psychological rehabilitation of preschool child with nonsyndromic oligodontia and anodontia in mixed dentition stage through conservative systematic approach: a case report with 5-year follow-up. Contemporary Clinical Dentistry, Mumbai, 2016; 7(2): 232-235.

20. RODRIGUES FV, et al. Aesthetic and functional rehabilitation in pediatric dentistry patient: a case report. Journal of Health Sciences, Londrina, 2019; 21(1): 77-81.

21. SALES PSM, et al. Reabilitação protética na dentição decídua. Archives of Health Investigation, São Paulo, $2018 ; 7(4)$ : 110.

22. SILVA BCM, et al. Importância da reabilitação oral através da prótese parcial removível: relato de caso. Revista Organizações em Contexto, São Bernardo do Campo, 2017; 1(2): 71-81.

23. SILVA, WSA, et al. Lesão central de células gigantes: relato de caso. Revista Eletrônica Acervo Saúde, São Paulo, 2019; 17(17): 01-09.

24. SHIBAYAMA R, et al. Reabilitação protética de paciente maxilectomizado - relato de caso. Revista Odontológica de Araçatuba, São Paulo, 2016; 37(2): 09-16.

25. SOUSA JM, et al. Utilização de prótese parcial fixa modificada na primeira infância: relato de caso. Odontologia Clínico-Científica, Recife, 2012; 11(3): 253-257.

26. SOUZA JEA, et al. Prótese parcial removível overlay: fundamentos clínicos e relatos de caso. Robrac, Goiânia, 2009; 18(47): 4148.

27. TRENTO CL, et al. Lesão de células gigantes central: relato de caso. Revista de Cirurgia e Traumatologia Buco-Maxilo-Facial, Camaragibe, 2009; 9(4): 39-44.

28. VALLE CN, et al. Carcinoma espinocelular oral: um panorama atual. Revista de Patologia do Tocantins, Tocantins, 2016; 3(4): 82102.

29. VULIĆEVIĆ, Z. et al. Prosthetics in paediatric dentistry. Balkan Journal of Dental Medicine, Belgrade, 2017; 1(1): 78-82. 